\title{
Quel rapport entre la métacognition et la performance à l'écrit?
}

Analyse de la situation d'étudiants en sciences humaines

Dyanne Escorcia

\section{OpenEdition}

Journals

\section{Édition électronique}

URL : https://journals.openedition.org/educationdidactique/870

DOI : $10.4000 /$ educationdidactique. 870

ISBN : 978-2-7535-1625-0

ISSN : $2111-4838$

\section{Éditeur}

Presses universitaires de Rennes

\section{Édition imprimée}

Date de publication : 15 décembre 2010

Pagination : 63-82

ISBN : $978-2-7535-1301-3$

ISSN : 1956-3485

\section{Référence électronique}

Dyanne Escorcia, "Quel rapport entre la métacognition et la performance à l'écrit ? ", Éducation et didactique [En ligne], 4-3 | Décembre 2010, mis en ligne le 10 décembre 2012, consulté le 16 août 2022. URL : http://journals.openedition.org/educationdidactique/870 ; DOI : https://doi.org/10.4000/ educationdidactique. 870 


\title{
QUEL RAPPORT ENTRE LA MÉTACOGNITION ET LA PERFORMANCE À L'ÉCRIT ? : Analyse de la situation d'étudiants en sciences humaines
}

\author{
Dyanne Escorcia (Théodile-CIREL, Université Lille 3
}

\begin{abstract}
Résumé :Ce texte présente les résultats d'une recherche dont le but était de déterminer le rapport entre les processus métacognitifs et la performance des étudiants dans le domaine de la production d'écrits. Nous avons conduit deux enquêtes à l'aide de questionnaires et d'entretiens d'explicitation. Cinquante-sept étudiants de psychologie et des sciences de l'éduction de l'Université Paris Ouest-Nanterre La Défense ont participé à ces dispositifs. Les résultats obtenus nous mènent à relativiser l'hypothèse selon laquelle les processus métacognitifs ont un rapport fort et positif avec la performance. Nos analyses mettent en évidence l'importance des connaissances métacognitives et de la capacité des étudiants d'utiliser ces informations afin de planifier et de guider leurs productions écrites.
\end{abstract}

Mots clés : metacognition, étudiants, écriture, connaissances métacognitives, autorégulation

Dyanne Escorcia

Le point de départ de cette recherche est la problématique d'échec des étudiants français en premiers cycles. Parmi les divers facteurs, individuels et sociaux, associés aux difficultés de réussite de cette population, notre travail s'intéresse à des variables cognitives, en particulier au fonctionnement métacognitif des étudiants en ce qui concerne la production d'écrits à l'université. Tout d'abord, nous évoquerons notre problématique et notre question de recherche, avant de présenter les aspects théoriques clés qui serviront à nos analyses. La démarche méthodologique sera ensuite explicitée, tout en précisant les outils utilisés et les échantillons à l'étude. Les résultats les plus marquants seront suivis d'une discussion pour, enfin, énoncer les conclusions principales de notre travail.

\section{Problématique d'échec des étudiants}

La question de l'échec des étudiants est, depuis quelques années, au cœur des dispositifs d'aide mis en place dans les universités françaises. Dans ce contexte, $59 \%$ des étudiants qui commencent leurs études arrivent à les terminer, soit 11 points de moins que la moyenne des pays de l'OCDE (OCDE, 2004). Selon une étude menée en 2001, la probabilité de réussite en L3 était de 75,4\% en trois ans dans l'ensemble des universités françaises ${ }^{1}$, pourcentage variable selon les filières. En 2005, les étudiants des
Sciences humaines avaient un taux de réussite du DEUG (actuelles L1 et L2) en deux ans de 50,8 \% et celui de L3 en un an était de $64 \%{ }^{2}$. Diverses études (Galand, Neuville, \& Frenay, 2005; Gruel, 2003) ont mis en lumière des facteurs individuels et sociaux pour expliquer l'échec des étudiants: la progressive massification de la population étudiante, les conditions matérielles de la vie, le contexte familial et socioculturel, la trajectoire scolaire, le manque de méthodes de travail, l'apprentissage du métier d'étudiant, entre autres.

Parmi les variables qui participent à l'échec à l'université, notre recherche s'intéresse plus précisément à la situation des étudiants dans le domaine de la production écrite. Pollet (2001) montre que les écrits de cette population traduisent un certain nombre de difficultés: non production du type de texte demandé, juxtaposition d'informations, centration sur des aspects lexicaux-sémantiques, faible organisation et absence de prise en compte des objectifs et du lecteur. D'autres obstacles, mis en lumière par Piolat \& Roussey (1996), indiquent que, lors de la réalisation de brouillons, les étudiants privilégient la mise en texte linéaire et la prise en compte des contraintes lexicales et syntaxiques dès le début de leurs démarches; par conséquent, ces sujets accordent peu d'attention à la confrontation d'idées sur le plan du contenu et écrivent ce qui leur vient à l'esprit par des associations locales. Il convient de signaler que 
nous ne porterons pas nos analyses sur les produits des étudiants, mais sur certains processus psychologiques qui participent à leurs démarches d'écriture et qui peuvent être liés à la production de textes réussis à l'université. C'est ainsi que notre objectif principal est d'identifier le rapport entre les dimensions métacognitives et les performances écrites des étudiants.

Par métacognition nous entendons, avec Flavell, Millet, \& Miller (1993, p. 255), « un ensemble de connaissances qui prennent la cognition comme objet et qui régulent un aspect quelconque du fonctionnement cognitif $»$. La question du lien entre la métacognition et la performance en écriture nous intéresse car même si les premières définitions de la métacognition apportées par Flavell (1992) insistent sur l'importance des composantes métacognitives pour la réussite dans divers domaines scolaires, les résultats de plusieurs recherches (Peronard, 2001; Mongeau \& Hill, 1998; Schraw, 2001) divergent quant à la corrélation des connaissances métacognitives et des stratégies d'autorégulation avec les performances des sujets en plusieurs champs d'activité.

En ce qui concerne la production d'écrits, les conclusions d'études menées en contexte anglo-saxon semblent plus ou moins concordantes. Des recherches mettent en évidence les effets des métaconnaissances sur la qualité des écrits (Graham, Harris, \& Mason, 2005; Raphael, Englert, \& Kitschner, 1989) ainsi que le rapport entre certains mécanismes d'autorégulation et la performance (Zimmerman \& Martinez-Pons, 2004; Graham, Harris, \& Troia, 1998; Zimmerman \& Risemberg, 1997). Nous n'avons pas recensé de recherches en France qui interrogent précisément ces rapports, ce qui nous amène à plusieurs questions: S'intéresse-t-on suffisamment à ce que les étudiants connaissent d'eux-mêmes, de la tâche et de leurs stratégies en matière d'écriture? Connaît-on la manière dont ils régulent leurs productions écrites? Sait-on comment les amener à améliorer leurs performances dans ce domaine? Les dispositifs d'aide s'appuientils suffisamment sur ce que l'on sait du fonctionnement des étudiants? Malgré le nombre important de recherches portant sur les facteurs associés à l'insuccès de ce groupe de sujets, rares sont les recherches qui s'intéressent à ce que ces derniers savent et savent faire, à leurs stratégies d'apprentissage, d'écriture et de lecture, à leurs méthodes de travail (Coulon, 1997; Fave-Bonnet \& Clerc, 2001; Romainville, 2002). En outre, il nous semble que tenter de répondre à ces questions est en cohérence avec les principes d'une didactique de l'écriture centrée sur l'élève, perspective qui, comme le précise Barré-De Miniac (2000), cherche l'accompagnement des processus d'apprentissage de l'écriture à travers la mise en lumière des particularités du scripteur et de son évolution.

À partir de la description des compétences métacognitives dans le domaine de la production d'écrits et de l'analyse du rapport entre ces caractéristiques et la performance des étudiants, nous cherchons à identifier quels éléments métacognitifs sont davantage associés à la réussite des étudiants en matière d'écriture. Notre hypothèse principale est que les individus qui obtiennent les meilleurs rendements dans la production d'écrits font preuve de niveaux plus élevés de connaissances métacognitives (portant sur eux-mêmes, la tâche et leurs stratégies) et de stratégies d'autorégulation. Par l'emploi du terme connaissances métacognitives nous établissons une différence avec les connaissances métaprocédurales. Les premières évoquent pour nous les informations du sujet qui résultent de son activité réflexive relative à son fonctionnement cognitif. Les connaissances métaprocédurales, en revanche renvoient, d'après Brassart (2005), à des informations portant sur les procédures cognitives mises en place par des sujets, ou plus précisément à « des représentations sociales ou - ethno-méthodes - qui circulent dans un groupe social, voire les théories ou modèles scientifiques plus ou moins vulgarisés $»$ (p. 102). Elles correspondent à des indications communiquées par un sujet (adulte ou enfant) sur « comment faire pour » (Fijalkow, 1995). Nous distinguons également les connaissances métacognitives des connaissances métalinguistiques; celles-ci portent comme l'indique Brassart (2005), sur les objets langagiers et non sur les processus cognitifs impliqués dans l'activité du langage. Enfin, les connaissances métacognitives sont différentes des informations qui résultent de l'activité métadiscursive dans le sens où, suivant Brossard (1994), celle-ci porte sur le fonctionnement des discours et des situations de communication.

\section{Apports des recherches relatives au rapport entre la métacognition et la performance}

Les travaux conduits par Flavell (1992) sur la nature de la métacognition ont été suivis d'une masse 
considérable de recherches qui analysent les composants de cette notion. Cependant, depuis l'émergence du terme métacognition, il n'existe pas d'unanimité quant à sa définition au sein de la communauté scientifique. Plus récemment, il existe une tendance à préférer le mot « réflexivité », concept plus large qui comporte selon Wolf (2005), au-delà de la dimension essentiellement psychologique, des aspects sociologiques, anthropologiques et épistémologiques. Dans notre cas, nous nous appuyons sur une approche issue de la psychologie cognitive, fondamentalement axée sur les définitions initiales de Flavell et ses collaborateurs. Mais avant d'aborder la question du rapport entre la métacognition et la performance, voyons quelques problèmes qui peuvent expliquer le manque de convergente autour du concept de métacognition.

Un premier point est le lien qui est établi souvent entre métacognition et prise de conscience. Ainsi, Pinard (1992) souligne que la métacognition relève aussi bien de la capacité du sujet de réfléchir sur ses fonctions cognitives et de prendre en charge de manière consciente et délibérée son fonctionnement. Cette affirmation rejoint celle de Noël (1991) pour qui la métacognition correspond à la conscience nécessaire à la capacité individuelle de verbaliser l'action et de porter un jugement sur ses actions et connaissances lors du déroulement d'une activité. D'autres auteurs ont cependant souligné l'idée que métacognition et prise de conscience ne vont pas forcement ensemble. Wolf (2005) rappelle qu'une action autorégulée peut avoir lieu sans impliquer l'accès au champ de conscience, et qu'une connaissance métacognitive ne mène pas systématiquement le sujet vers un processus de verbalisation consciente ni vers la régulation de ses activités cognitives. Brown (1987) et Pinard (1986) avaient eux aussi soulevé la non dépendance entre la prise de conscience et les dimensions métacognitives. Bien que nous rejoignons ces derniers apports et acceptions que la métacognition comporte également une qualité non consciente, notre intérêt ici concerne exclusivement les processus qui peuvent être objet de verbalisation réfléchie de la part du sujet. En ce sens, la métacognition relève pour nous essentiellement de la capacité de l'individu de prendre son fonctionnement cognitif comme objet de réflexion et de s'appuyer sur ces informations afin de mener à bien son activité. C'est ainsi que les processus métacognitifs implicites - non conscients- ne seront pas objet de nos analyses.
Une autre raison du manque de convergence relativement à la définition du terme métacognition nous semble être le caractère composite accordé à ce concept. En effet, selon Schraw \& Sperling-Dennison (1994), la plupart des théories actuelles sur la métacognition déclinent la métacognition en deux dimensions qui sont les connaissances métacognitives, d'une part, et la régulation exercée par l'individu sur son apprentissage, d'autre part. Nous sommes d'accord avec Wolf (2005) qui ponctue le sens problématique du fait d'employer la même expression pour nommer deux processus qui peuvent s'avérer indépendants. Il nous semble toutefois que la prise en compte de la métacognition dans sa globalité est nécessaire et que le risque de confusions est minimisé grâce à l'apport de définitions clarifiées de chaque composante et à l'analyse des diverses modalités du rapport entre ces dimensions. Ce choix répond ainsi à notre projet d'éluder une compréhension partielle de ce qui se joue dans la métacognition. En ce sens, nous rejoignons Boekaerts (1999) pour qui la métacognition implique l'interaction de connaissances préalables qui informent le sujet sur les objectifs de la tâche et l'aident à générer de plans d'action et à sélectionner les stratégies les plus adéquates en fonction des buts et du contexte. En d'autres termes, l'analyse des deux composantes nous paraît pertinente car, comme le proposent Mongeau \& Hill (1998), la métacognition comprend aussi bien la capacité individuelle d'expliciter ses stratégies d'apprentissage que la mise en place d'une démarche suffisamment anticipée, en se fixant des objectifs et en corrigeant son processus au fur et à mesure de l'apprentissage.

Après avoir abordé ces divergences, prenons par la suite chacune des composantes métacognitives afin de les définir et d'évoquer leurs liens avec la performance. En ce qui concerne les connaissances métacognitives, ces informations renvoient à la compréhension, par l'individu, de ses fonctions cognitives. Il s'agit de croyances relatives aux variables qui affectent le déroulement de l'activité cognitive. Selon Brown (1987), les connaissances métacognitives peuvent être de plusieurs sortes: déclaratives ou personnelles (référées à la tâche ou à l'individu lui-même, à ses capacités et faiblesses); procédurales ou stratégiques (qui font allusion aux procédures et aux méthodes personnelles); ou conditionnelles (qui renseignent l'individu à propos de la relation entre ses stratégies et les caractéristiques de la tâche). Ces divers types 
de connaissances métacognitives ne sont pas qualitativement différents d'autres sortes de connaissances stockées en mémoire à long terme (Flavell, 1987). En effet, elles peuvent être relativement stables, fixes, fausses et susceptibles d'utilisation automatique ou délibérée. En particulier, les connaissances métacognitives relatives à la tâche (type de texte, caractéristiques et contraintes de l'écrit à réaliser, conditions de réalisation) renferment, quant à la production écrite, des connaissances métatextuelles car, comme l'expriment Coutelet \& Rouet (2004), ces dernières se rapportent aux caractéristiques des écrits et à leurs fonctions. Ces informations que l'individu possède à propos des textes en général, de leur structure, deviennent des connaissances d'ordre métacognitif à partir du moment où elles constituent des objets de réflexion et/ou de jugement en fonction des conditions spécifiques de l'activité et des connaissances personnelles du sujet portant sur ses stratégies.

Quant au lien entre les connaissances métacognitives et la performance en plusieurs champs d'activité, Flavell (1992) a supposé que cette composante métacognitive joue un rôle important dans le choix, l'évaluation, l'abandon des buts et des stratégies pendant l'action. Pour Melot \& Corroyer (1992), l'aspect régulateur de l'action des métaconnaissances contribue à l'efficacité des individus. Ce lien a été nuancé par plusieurs travaux, comme celui de Mongeau \& Hill (1998), qui constatent que les métaconnaissances ne sont pas forcement une caractéristique inhérente aux sujets les plus performants. Ces auteurs, qui ont mené une étude auprès d'étudiants apprenant l'anglais comme langue seconde, ont montré qu'il n'existe pas de corrélation linéaire entre les métaconnaissances et les scores obtenus aux tests d'anglais.

Cependant, dans le domaine de l'écriture, certains travaux mettent en évidence qu'il existe un lien fort et positif entre les connaissances métacognitives et la performance. Ferrari, Bouffard, \& Rainville (1998) analysent les connaissances relatives à la tâche chez des rédacteurs plus et moins performants. Ces données révèlent que le premier groupe de sujets possède des connaissances plus précises quant aux contraintes de l'écrit et par rapport aux principales attentes du lecteur. De la même manière, Piolat \& Roussey (1991a) montrent l'existence de niveaux plus élevés de connaissances métacognitives chez des scripteurs experts; cette caractéristique, expli- quent les auteurs, rend possible l'autoévaluation efficace des écrits et leur modification en regard des buts lorsque cela est nécessaire. Englert \& Kirschner (1989) trouvent que les scripteurs les plus expérimentés comprennent davantage les stratégies d'écriture et reconnaissent plus aisément les divers processus rédactionnels. Enfin, Victori (1999) met en évidence l'idée que les rédacteurs ayant produit des écrits dont la qualité est jugée comme étant plus faible explicitent des connaissances peu justes par rapport aux caractéristiques du type d'écrit qu'ils ont produit.

Pour comprendre la métacognition, il est également nécessaire de bien distinguer ce qui est pour nous l'autorégulation. Voyons d'abord les éléments que ce concept recouvre. Selon Schraw (2001) la plupart des recherches s'accordent autour de l'identification de trois catégories de processus d'autorégulation: la planification (la sélection de stratégies et l'adjonction des efforts nécessaires à l'action); le contrôle (le suivi individuel du déroulement de sa propre démarche); et l'évaluation (l'estimation des résultats et de l'efficacité des comportements mis en œuvre). Au regard de la théorie sociocognitive, Zimmerman (2002) conçoit ces stratégies en tant que phases d'un processus cyclique orienté vers la surveillance et la gestion autonome du fonctionnement cognitif. Lautorégulation serait alors un ensemble de mécanismes autodirigés qui rendent possible la transformation du cours de l'action grâce à la relation cyclique de facteurs personnels, comportementaux et environnementaux (Zimmerman, 2002). Cet auteur identifie plusieurs stratégies telles que la fixation de buts, l'autoévaluation, la production d'images mentales, la gestion du temps, l'autoinstruction, l'utilisation de guides ou de modèles ainsi que l'arrangement du contexte.

Zimmerman \& Martinez-Pons (1986) ont mis en place une étude appuyée sur des entretiens structurés afin d'examiner le rapport entre les stratégies d'autorégulation et la réussite dans plusieurs contextes d'apprentissage (la maison, les cours, la préparation de devoirs). Ces auteurs ont trouvé: a) que les sujets ayant obtenu des résultats faibles ont explicité un nombre plus élevé de stratégies non régulées et b) qu'il existe un rapport fort entre les variables analysées. Ce lien est plus remarquable en ce qui concerne les stratégies de recherche d'aide, d'autoinstruction 
et d'organisation. En revanche, la relation ne paraît pas être linéaire entre l'autoévaluation et la réussite.

Dans l'ensemble des stratégies d'autorégulation citées, la fixation de buts, l'autoévaluation et l'autoinstruction vont nous intéresser par la suite. Ces méthodes ont été particulièrement étudiées en contexte anglosaxon, au regard de l'approche sociocognitive, et dans divers domaines d'activité. Plus précisément, Zimmeman \& Kitsants (1997) analysent les buts et les critères d'évaluation en fonction de l'objet sur lequel ils portent. Ces auteurs constatent notamment que les objectifs axés sur le produit à réaliser (les caractéristiques de l'écrit) sont moins corrélés avec la performance que les buts privilégiant le processus (objectifs focalisés sur le choix, la gestion ou la correction des méthodes mises en œuvre).

Quant à l'autoévaluation, Graham, Harris, \& Troia (1998) constatent que cette stratégie permet à l'individu de maintenir un niveau de concentration élevé, de déduire l'efficacité des méthodes employées et de se rendre compte des difficultés éprouvées. De leur côté, Zimmerman \& Risemberg (1997) n'ont pas constaté de rapport positif entre l'autoévaluation et la réussite. Ces auteurs ont trouvé que les rédacteurs qui s'autoquestionnent par rapport à leurs critères d'autoévaluation acquièrent un niveau plus conséquent de métaconnaissances, mais ils n'améliorent pas nécessairement la qualité des écrits produits.

Enfin, l'autoinstruction, qui consiste en l'utilisation de dialogues internes, subvocales ou à haute voix, pour autoréguler le comportement (Zimmerman \& Kitsantas, 1997), serait une stratégie utile notamment durant la révision des écrits car elle augmenterait, chez l'individu, la surveillance et la détection d'erreurs. Zimmerman \& Risemberg (1997) ont trouvé que des élèves qui lisaient à haute voix leurs textes, en s'interrogeant de manière réitérée sur la qualité de ces derniers, ont détecté davantage d'erreurs. Les mécanismes par lesquels ces formes de contrôle de l'activité sont utiles lors de la révision d'écrits ont été analysés en contexte français par Piolat $\&$ Roussey (1991b). Lapport de ces auteurs consiste en expliquer que cette sorte d'autoverbalisation rend le scripteur capable de s'interroger sur l'adéquation entre ses objectifs et son produit pour ensuite mettre en place des améliorations. Piolat \& Roussey (1991b) soulèvent ainsi l'importance d'avoir des objectifs clairs: il s'agit d'informations qui constituent une source importante d'autorégulation notamment lorsque le scripteur vérifie la qualité de son écrit.

Les recherches dont nous venons de rendre compte montrent que les rapports entre les composantes métacognitives et la réussite dans la production d'écrits sont variables, notamment en ce qui concerne les stratégies d'autorégulation. Remarquons également que les études présentées se focalisent sur l'une ou l'autre composante de la métacognition. Dans le cadre de cette recherche, le choix d'explorer simultanément les connaissances métacognitives et les stratégies d'autorégulation correspond au caractère bidimensionnel de la métacognition que nous avons évoqué précédemment. En accord avec les auteurs que nous avons convoqués ici (Flavell, 1992; Boekaerts, 1999; Mongeau \& Hill, 1998) pour définir la métacognition, nous pensons que si l'on n'étudie que les connaissances, peu de renseignements seront obtenus sur le rôle de ces informations dans l'action. De la même manière, si l'on se limite à l'analyse des stratégies, seraient ignorées les connaissances qui appuient les prises de décisions et les choix stratégiques.

\section{Démarche méthodologique}

Afin de mener notre recherche, nous avons mis en place deux dispositifs suivant des approches méthodologiques différentes: l'une qualitative-dépendante et l'autre quantitative-indépendante. Pour déterminer le caractère dépendant ou indépendant de la méthode nous nous appuyons sur la classification établie par Mariné \& Huet (1998). Dans le premier cas, nous avons utilisé des entretiens d'explicitation; ce dispositif est dépendant car notre interrogation porte sur une activité précise dans laquelle les sujets avaient participé auparavant. La seconde méthodologie emploie un questionnaire construit dans le cadre de cette recherche. Le caractère indépendant du dispositif résulte du fait que les items de l'instrument ne renvoient pas à une activité qui s'est déroulée à un moment déterminé mais plutôt à une description des pratiques habituelles d'écriture. Ces choix méthodologiques répondent également à notre intérêt d'étudier non seulement ce que les étudiants connaissent de leur fonctionnement en écriture mais aussi ce qu'ils font de ces informations en tant que scripteurs dans une situation particulière. Il résulte également 
pertinent de souligner que, dans les deux démarches, il s'agit de connaissances métacognitives et de stratégies d'autorégulation telles qu'elles sont déclarées par les sujets et non de processus métacognitifs effectivement pratiqués par les individus.

\section{Description des dispositifs de recherche: échantillons et déroulement des enquêtes}

\section{Premier dispositif}

Nous avons conduit des entretiens d'explicitation auprès de douze étudiants de première année en sciences de l'éducation (Université Paris OuestNanterre La Défense, année 2004-2005). Le choix d'étudier ce groupe de sujets nous a paru cohérent avec la problématique de notre recherche. Ces étudiants possédaient l'une des caractéristiques associées aux difficultés de réussite en contexte universitaire, à savoir la diversité de publics en termes d'âge, de formations et de parcours. En effet, le programme d'études dont il est question ici prévoyait un dispositif particulier pour faciliter l'entrée des étudiants dans le champ des sciences de l'éducation. Nous avons pu confirmer la variété des profils de ces étudiants lors de la phase exploratoire de notre recherche. Enfin, l'intérêt d'analyser la situation de ces sujets a été conforté par le faible taux de réussite qu'ils partagent avec l'ensemble des étudiants en Sciences humaines de leur Institution, pourcentage qui était de $49 \%$ en 2005, l'un de plus faible au niveau national selon une étude de la DEP ${ }^{3}$.

Afin de constituer notre échantillon, nous avons contacté un groupe de 40 étudiants qui avaient produit récemment des fiches de lecture dans le cadre d'une unité d'enseignement. Leurs écrits ont été notés par l'enseignant responsable du cours sur un coefficient de 20 mais nous nous sommes abstenue d'apprendre ces résultats et de les intégrer à notre dispositif de recherche. Les performances écrites des étudiants ont, en revanche, été déterminées dans notre étude à l'aide d'une grille élaborée par nos soins, instrument qui nous a permis de recueillir les avis de plusieurs évaluateurs par rapport à des critères communs et clarifiés (cf. section description des outils). Bien que consciente du fait que les enjeux de l'évaluation liés au processus d'enseignement aient pu intervenir d'une certaine manière lors de la réalisation de la tâche, notamment en termes de motivation et d'expectatives des étudiants, nous n'avons pas fait de ces implications un axe d'analyse. Toutefois, nous avons mené les entretiens avant que les étudiants ne prennent connaissance de leurs notes attribuées par l'enseignement responsable; nous voulions ainsi affecter le moins possible les perceptions et représentations des sujets relatives à leurs produits et à leurs processus mis en place.

Au total 12 sujets ( 10 filles et 2 garçons) âgés de 17 à 39 ans (âge médian de 24 ans) ont accepté de participer aux entretiens. Il leur a été demandé de décrire la manière dont ils avaient rédigé leurs fiches de lecture. La distance temporelle entre la réalisation des écrits et les interviews a été variable (de 4 à 5 semaines) et déterminée en partie par les emplois du temps des étudiants. Ce délai nous a paru convenable suite à des résultats d'expérimentations préalables que nous avons réalisées durant la phase exploratoire de notre recherche (Escorcia, 2007). En effet, nous avons testé deux types de dispositifs: d'abord, des entretiens au cours desquels les sujets décrivaient des démarches de rédaction qu'ils avaient menées quelque temps auparavant; un second dispositif consistait en des entretiens réalisés tout de suite après la production d'écrits. Nous avons constaté que le premier procédé posait des problèmes de rappel aux étudiants, et que le second induisait des entretiens très courts et superficiels ainsi que des écrits de faible qualité (souvent abrégés, des simples énumérations d'idées, ensembles déstructurés). De fait, cet exercice imposait aux étudiants une double contrainte: écrire tout en gérant les charges cognitives impliquées dans l'écriture, puis devoir s'exprimer, au cours d'entretiens, à propos de leurs activités immédiatement après celle-ci. Nous avons conclu que le dispositif le plus pertinent était celui de mener des interviews quelques semaines après la production, et d'organiser ces échanges autour de deux phases clés: l'une de description du déroulement afin de tracer les grandes étapes de la production, l'autre d'explicitation en donnant la possibilité de choisir des moments à décrire. Cette méthode s'est montrée propice à la description des moments marquants pour le sujet, où l'expérience subjective d'explicitation et d'élucidation de l'action faisait appel à sa mémoire concrète. Cette condition, qui constitue selon Vermersch (2004) un point central pour faciliter l'explicitation, a été significative et nous a permis, en même temps, de contourner les difficultés de rappel chez les sujets. 


\section{Second dispositif}

Cette partie de la recherche s'appuie sur les données d'un questionnaire passé auprès de 45 étudiants de L3 en Psychologie (Université Paris Ouest-Nanterre La Défense, 2005-2006). Tout comme dans le premier dispositif, nous avons tenu compte du fait que ce groupe de sujets intégrait une population sensible à l'échec. Plus globalement, dans certaines universités française, les étudiants de psychologie montrent des taux de réussite très bas, soit moins de $40 \%$ (Jarousse \& Michaut, 2001). De plus, la production d'écrits semble leur poser des problèmes comme l'a constaté Mucchielli (1998): difficultés à mettre en relation la théorie et la pratique, ainsi qu'élaboration de contenus perçus comme étant éloignés du concret.

Les étudiants interrogés avaient de 18 à 32 ans (38 filles et 7 garçons) et présentaient un âge médian de 20 ans. Ces sujets participaient à un enseignement d'initiation à la recherche dans lequel ils devaient participer à une expérimentation, en tant que sujets, parmi plusieurs dispositifs proposés. Les étudiants qui ont choisi notre étude ne connaissaient que le titre, la date et l'heure à laquelle ils seraient convoqués pour l'expérience. Lors d'une matinée, nous leurs avons proposé de rédiger une dissertation contenant une réflexion personnelle et argumentée par rapport à un thème imposé. Les étudiants savaient que leurs écrits seraient évalués exclusivement dans le cadre de la recherche et que ces évaluations n'auraient aucune incidence sur leurs notes dans le cadre de leurs études. À la fin de la rédaction (une heure leur a été donnée), les étudiants ont rempli un questionnaire (Escorcia, 2007) qui cherchait à déterminer leurs niveaux de métacognition. Contrairement au premier dispositif, le temps écoulé entre la production d'écrits et le questionnement est dans ce cas très court; mais cela ne nous a pas paru problématique, en ce qui concerne les efforts liés à la description des pratiques personnelles, le questionnaire étant de ce point de vue moins exigeant que l'entretien d'explicitation.

Par ailleurs, nous avons estimé que le fait d'administrer le questionnaire tout de suite après la tâche n'était pas incompatible avec le caractère " indépendant » de notre second dispositif car, comme nous l'avons dit, cet instrument n'interro- geait pas précisément les étudiants par rapport à une situation d'écriture particulière. Même si les sujets pouvaient avoir tendance à répondre par rapport à leurs productions les plus récentes, en particulier l'écrit produit durant le dispositif, ils pouvaient également évoquer d'autres moments d'écriture qui seraient davantage représentatifs de leurs conditions habituelles de production et de leurs manières de travailler. En bref, bien que la proximité entre la situation vécue et la production des dissertations ait pu affecter les réponses des étudiants, ces dernières ont pu aussi être influencées par leurs représentations d'autres expériences d'écriture probablement plus en accord avec leurs situations d'étudiants. En ce sens, le caractère indépendant du dispositif n'était pas remis en question.

Après la production des écrits et la passation des questionnaires, les textes des étudiants ont été notés par des enseignants extérieurs à la recherche suivant la grille d'évaluation que nous avons construite et réadaptée au regard des caractéristiques de l'écrit en question.

\section{Outils}

Les entretiens d'explicitation avaient pour objectif d'aider les étudiants à décrire le plus finement possible leurs démarches d'écriture. Nous les amenions à expliciter les diverses étapes de leurs activités en essayant de leur faire évoquer leurs connaissances et leurs manières de faire. Sans compter sur une grille de questions préétablie, nos questionnements étaient toujours du même type: qu'est-ce que tu as fait pour commencer? Et ensuite? Et quand tu faisais cela que faisais-tu précisément? Par quoi as-tu terminé ? Comment as-tu reconnu que c'était fini? Les contraintes de rappel imposées par ce type de questions pouvaient rendre difficile l'accès aux détails de leurs démarches, provoquant ainsi des descriptions générales et des explications plutôt que des véritables explicitations au sens proposé par Vermersch (1994). Afin de minimiser ses impasses, les deux phases des entretiens déjà décrites (section précédente) nous permettaient d'obtenir un accès progressif à la mémoire grâce à la fragmentation de la situation vécue en ses grandes étapes, d'une part, et à la description de plus en plus fine des moments choisis par le sujet, d'autre part; cette explicitation 
était facilitée par l'évocation d'aspects sensoriels et par le rappel réitéré du contexte de l'action précise.

Par ailleurs, afin d'élaborer la grille utilisée pour estimer la variable « performance à l'écrit » nous avons mené des entretiens auprès d'enseignants en sciences humaines et sociales de l'Université Paris Ouest-Nanterre La Défense (Escorcia, 2007). Nous avons demandé à ces derniers de décrire les critères d'évaluation de textes qu'ils utilisaient pour noter les devoirs écrits des étudiants (enquête menée en 20042005). Cette démarche nous a permis de repérer et de regrouper les critères des enseignants en cinq catégories: aspects linguistiques, capacité d'analyse, éléments d'argumentation, appropriation et mise en relation des connaissances, respect de la consigne, présentation de l'écrit. Dans le cadre du premier dispositif, les évaluateurs ont été trois enseignants qui n'intervenaient pas au sein de l'enseignement suivi pas les étudiants ni faisaient partie de notre recherche. Ils devaient noter de 0 à 3 leur degré d'accord avec les affirmations contenues dans la grille (annexe 1, grilles utilisées dans les deux dispositifs).

Puisque toute évaluation implique un certain degré de subjectivité (Hadji, 1997), nous avons choisi de valider cette mesure de la performance écrite par la triangulation d'observateurs et par l'utilisation d'une grille de critères communs et établis à l'avance. L'évaluation critériée a en effet l'avantage de fournir des référents clairs pour apprécier la réussite (Hadji, 1997) et de contrôler ainsi certains biais liés à la subjectivité des évaluateurs. Par ailleurs, nous avons assuré la validité de cet instrument de mesure lors de sa construction en respectant deux conditions citées par Maxwell (1999): d'abord, en nous accordant aux significations exprimées par les enseignants interviewés, sans apporter nos préjugés; puis en recueillant la totalité des critères évoqués. Nous devons néanmoins considérer certains limites de l'évaluation criteriée comme par exemple le fait que la subjective n'est pas éliminée complètement; elle a pu intervenir notamment en fonction des niveaux d'exigence de chaque enseignant-évaluateur, de leurs conceptions de l'écriture, de leur niveau de fatigue lors de la tâche. D'autre part, cette mesure de la performance est spécifique par rapport à notre dispositif. Cela veut dire que les mêmes étudiants peuvent se montrer plus ou moins performants, produire des meilleurs écrits ou des documents moins bons dans d'autres conditions d'expérimentation ou même dans le cadre de leurs enseignements. Il ne s'agit par conséquent d'une mesure absolue de la performance écrite, elle est ainsi circonstanciée.

Le second outil utilisé est un questionnaire pour explorer la métacognition (annexe 2, questionnaire). Cet instrument se compose de deux sous-échelles, l'une mesure les connaissances métacognitives et l'autre l'autorégulation; ces sous-échelles possèdent des bonnes consistances internes (alphas de. 74 et. 70 respectivement). Plus précisément, nous évaluons trois types de métaconnaissances (relatives à la tâche, aux faiblesses/points forts, et aux stratégies du scripteur) et six stratégies d'autorégulation (fixation de buts, autoinstruction, réalisation de plans, les critères d'autoévaluation, l'utilisation de modèles et l'arrangement du contexte). Les étudiants devaient citer la fréquence avec laquelle les caractéristiques citées faisaient partie de leurs pratiques d'écriture, en utilisant une échelle à quatre points qui allait de « jamais » à « tout le temps » en passant par « rarement » et « fréquemment ».

\section{Méthode d'analyses des données}

Les entretiens ont été transcris dans leur intégralité puis nous avons fait des analyses de contenu avec le but de décrire le fonctionnement des étudiants du point de vue des informations qu'ils ont exprimées concernant leurs connaissances et leurs stratégies mises en œuvre. Les catégories d'analyses utilisées ont porté sur trois types de métaconnaissances (personnelles, stratégiques et conditionnelles) et trois processus d'autorégulation (planification, contrôle et auto-évaluation). Au-delà des analyses qualitatives, les items (phrases prélevées des entretiens) classés dans les catégories ont été comptabilisés puis leur nombre a été saisi dans le logiciel SPSS afin d'établir des corrélations avec les notes obtenues par les étudiants dans leurs écrits (test Spearman « rhô »). Le logiciel SPSS a également été utilisé pour mener des traitements statistiques à partir des résultats des questionnaires dans le but de corréler le niveau des processus métacognitifs et la qualité des écrits (corrélation BravaisPearson « $\mathrm{r} »)$. Compte tenu du nombre réduit de sujets, nos analyses n'ont pas l'intention de généraliser les résultats mais de nous construire une idée de la situation du groupe en question. 


\section{Résultats}

\section{Premier dispositif: entretiens d'explicitation}

À partir des entretiens analysés, nous avons obtenu des informations relatives à ce que les étudiants connaissent (d'eux-mêmes, de la tâche et de leurs stratégies d'écriture) et à ce qu'ils font (processus d'autorégulation). Un premier point qui se dégage de ces analyses est que les connaissances relatives à eux-mêmes en tant que scripteurs font référence principalement à leurs faiblesses dans ce domaine. Les processus cognitifs par rapport auxquels ils croient posséder des difficultés concernent essentiellement la construction de phrases, la recherche de mots et le vocabulaire. Si nous tenons compte des opérations de planification, mise en texte et révision décrites par Hayes et Flower (1980), nous pouvons dire que les difficultés déclarées par les sujets renvoient en particulier aux aspects formels de la langue écrite que le scripteur doit gérer à tout moment de sa production.

Par ailleurs, la lecture des interviews nous a permis de noter que les connaissances des étudiants à propos des caractéristiques de l'écrit mettent en évidence leurs représentations des exigences textuelles d'une fiche de lecture ${ }^{4}$. D'une part, ils définissent ce qui est un compte rendu de lecture en soulignant le caractère descriptif de ce type d'écrit. D'autre part, les interviewés ont une représentation claire de la partie « analyse critique » que l'enseignant avait demandée à produire dans la fiche de lecture. C'est alors qu'ils reconnaissent plusieurs contraintes de la partie analyse: lecture approfondie des sources bibliographies, extraction des idées principales à partir des textes, expression de leurs points de vue personnels. Nous remarquons que l'élaboration de ces deux segments de l'écrit en fonction des caractéristiques textuelles déclarées par les étudiants ne semble pas leur avoir posé de difficultés; ils n'ont pas désigné ces exigences lors de la description de leurs faiblesses présentées dans le paragraphe précédant.

Un autre type de métaconnaissances porte sur des informations relatives aux stratégies employées durant les phases de l'activité scripturale. Quant à la planification de leurs écrits, les étudiants déclarent avoir mis en œuvre une variété importante de méthodes. Ainsi, la récupération d'idées est marquée par la prise de notes. De même, l'élaboration de plans et la construction de brouillons sont des méthodes qu'ils déclarent avoir privilégié durant l'organisation de leurs écrits. Lors de la mise en texte, certains étudiants scripteurs évoquent l'utilité de la relecture pour contrôler des opérations comme la recherche de mots et la construction de phrases. D'autres interviewés affirment avoir régulé leurs contextes en choisissant de textes modèles ou en aménageant l'endroit le plus favorable pour leurs productions. Enfin, la révision des écrits se caractérise par l'utilisation de verbalisations subvocales mises en place lors des relectures des écrits dans le but d'identifier des erreurs et d'en faire des diagnostiques.

Outre ces connaissances, nous avons analysé la manière dont les étudiants régulent leurs productions écrites à travers les stratégies de fixation de buts, d'autoévaluation et d'autoinstruction. En ce qui concerne la première, les objectifs émis par les étudiants concernent majoritairement le contenu à exprimer (suivant la classification proposée par Zimmerman [1998], entre buts de processus et de performance ou produit). Les sujets cherchaient, par exemple, à abréger les idées durant la mise en texte, ou à supprimer des informations lors de la correction de l'écrit. En revanche, peu d'étudiants avaient des objectifs qui rappellent la gestion des processus ou la manière dont ils souhaitaient mettre en œuvre les diverses opérations. Également, leurs méthodes d'évaluation portaient principalement sur les textes plutôt que sur la vérification ou l'ajustement de leurs processus au cours de l'action. Ils déclarent avoir évalué principalement des aspects linguistiques (clarté des idées, construction des phrases, mots employés) ainsi que la pertinence des idées exprimées. Cependant, nous ne constatons pas d'indices faisant référence à, par exemple, l'évaluation de l'efficacité de leurs stratégies de correction ou de réalisation de plans.

Par rapport à la stratégie d'autoinstruction, nous souhaitions savoir à quel moment de la rédaction étaient utilisées les verbalisations subvocales et quel était leur objet principal. C'est essentiellement durant la phase de mise en texte comme telle que les sujets déclarent avoir utilisé cette stratégie afin d'élaborer le contenu de l'écrit, d'organiser leurs idées, de choisir les mots et de construire des phrases. Quant aux objets de ces autoverbalisations, les étudiants ont 
déclaré avoir surveillé principalement l'élaboration des contenus de l'écrit et les aspects formels de la production. En bref, l'ensemble des stratégies analysées révèle que l'autorégulation à travers les buts, les critères d'autoévaluation et l'autoinstruction paraît centrée sur les aspects formels et les normes de l'écrit ce qui semble correspondre à l'importance que les étudiants accordent à ces éléments au moment d'évoquer leurs faiblesses en matière d'écriture.

Pour finir, la quantification des items retenus durant l'analyse des entretiens montre qu'il existe une association positive significative entre les connaissances métacognitives relatives aux stratégies et la performance (rhô $=.65 ; \mathrm{p}<.020$ ). Cela signifie que plus les étudiants produisent des textes de bonne qualité, plus ils évoquent des informations relatives à leurs stratégies et à leurs caractéristiques en tant que scripteurs. Mais il s'agit de la seule corrélation significative qui a été détectée suite à nos calculs statistiques, ce qui veut dire qu'il n'apparaît pas de rapport entre les variables performance et métacognition si nous entendons cette dernière comme à la fois les connaissances métacognitives et les stratégies d'autorégulation.

\section{Second dispositif: questionnaires}

Pour commencer, nous avons considéré l'ensemble des réponses issues du questionnaire. Il apparaît une relation forte entre la métacognition et la performance $(r=.45, \mathrm{p}<.002)$. En revanche, lorsque nous observons les résultats des corrélations par sous-échelle, nos résultats ne confirment que la dépendance entre les variables «performance » et « métaconnaissances », plus particulièrement en ce qui concerne les connaissances relatives à la tâche et aux capacités personnelles (items $5[\mathrm{r}=.327$; $\mathrm{p}<.028$ ] et $10 \mathrm{r}=.433 ; \mathrm{p}<.003]$ ). Cela veut dire que les étudiants qui estiment savoir organiser les informations avant de rédiger et ceux qui déclarent posséder des connaissances sur la structure du texte à écrire, ont produit des écrits jugés comment étant de meilleure qualité.

En ce sens, notre étude ne corrobore pas l'hypothèse d'une dépendance positive forte entre l'autorégulation et la capacité des scripteurs de produire de textes de qualité dans le contexte universitaire.
Cependant, si nous analysons individuellement chaque stratégie d'autorégulation, il est possible d'identifier certains items fortement corrélés avec la performance. Ainsi, la fixation de buts (item $1[\mathrm{r}=.375 ; \mathrm{p}<.011]$ ), la réalisation de plans (item $11[\mathrm{r}=.312 ; \mathrm{p}<.037]$ ) et l'autoinstruction (item $19 \mathrm{r}=.340 ; \mathrm{p}<.022$ ]) sont des indicateurs forts de la réussite. Autrement dit, les étudiants qui signalent avoir pour habitude d'identifier avant la tâche les informations à écrire et les méthodes à utiliser, ceux qui déclarent organiser leurs textes en hiérarchisant les idées, et ceux qui produisent et utilisent des informations pertinentes pour s'autoguider au cours de la production, ont écrit les textes les plus réussis.

\section{Discussion}

À partir des résultats des deux dispositifs nous arrivons à des constats différents quant au rapport entre la métacognition et la performance écrite des étudiants. Lanalyse des entretiens montre qu'au regard du score total des indices de métacognition enregistrés (à la fois les connaissances métacognitives et les stratégies d'autorégulation), il n'y a pas de rapport significatif avec la qualité des produits. En revanche, les données issues des questionnaires confirment l'hypothèse selon laquelle la métacognition déclarée (la somme des réponses à l'ensemble des items de l'instrument) a un rapport fort et positif avec la performance. Cet écart au niveau des résultats d'un dispositif à l'autre peut être expliqué par la nature même des processus métacognitifs mesurés dans chaque étude. Comme l'affirment Mariné et Huet (1998), lorsque l'on interroge la métacognition sans faire référence à une tâche particulière ( $2^{\mathrm{e}}$ dispositif dit «indépendant $\left.»\right)$, sont mesurées plutôt des connaissances métacognitives généralisables. Ainsi, d'après nos résultats, le rapport entre les deux variables est plus évident lorsque l'individu exprime de manière decontextualisée ses connaissances et ses manières d'agir, que lorsqu'il parle d'une tâche précise ( $1^{\mathrm{er}}$ dispositif dit « dépendant»).

Malgré ces différences, l'analyse du rapport de chaque composante métacognitive avec la performance nous apporte quelques données convergentes. Ainsi, à partir des deux dispositifs, nous constatons qu'en observant plus précisément les scores des 
métaconnaissances, il apparaît un rapport positif significatif avec la qualité des écrits. En ce sens, les étudiants qui ont déclaré, au cours des entretiens et à travers des questionnaires, un nombre plus important de connaissances relatives à la tâche, à eux-mêmes (leurs capacités et faiblesses) et à leurs stratégies, ont obtenu des notes à l'écrit plus élevées.

En ce qui concerne l'autorégulation, les résultats des deux études relativisent l'hypothèse d'un lien direct entre la qualité des écrits et l'ensemble des stratégies analysées. Seulement à partir des données du questionnaire il est possible de dégager certains éléments associés à la réussite. Ce qui compte est la capacité du sujet d'utiliser ses connaissances afin d'organiser et de guider son processus d'apprentissage en planifiant son activité grâce à l'identification de buts, et en contrôlant son processus par un suivi permanent (dialogues avec soi-même). Ces résultats confirment les conclusions de Zimmerman \& Martinez-Pons (1986) et celles de Zimmerman \& Kitsantas (1997). En ce qui est de l'autoévaluation nous rejoignons aussi le constat de Zimmerman \& Risemberg (1997) quant à l'absence de lien avec la performance.

Ce manque de rapport peut être expliqué, comme nous l'avons fait précédemment, par les différences entre les dispositifs: le lien entre les composantes paraît plus difficile à saisir lorsque les sujets font référence à une tâche particulière. Il ressort des entretiens une diversité importante de manières de procéder, raison pour laquelle nous aurions besoin d'un nombre plus important de sujets afin d'effectuer de nouveaux calculs de corrélation. Outre cette explication, nous pouvons penser, comme le proposent Zimmerman \& Kitsantas (1997), que les interviewés ne décrivent pas toutes leurs stratégies car ils n'en ont pas toujours conscience. Selon ces auteurs, les sujets performants n'ont pas besoin de contrôler consciemment le déroulement de leurs actions et, par conséquent, ils n'explicitent pas spontanément leurs stratégies au cours des entretiens. C'est la même hypothèse de Vignet (repris par J.-L. Pommet, 2005) quand il parle de «compétences incorporées » ou de savoirs complexes utilisés de manière automatique. Dans le cas de notre premier dispositif, nous pourrions supposer que les étudiants scripteurs n'ont pas évoqué l'éventail de leurs stratégies soit parce qu'ils n'en ont pas conscience, soit parce qu'ils ne l'ont pas utilisé entièrement dans la production en question. Afin de valider cette supposition, il faudrait mener des observations directes des démarches de scripteurs. Ainsi, nous pourrions constater les stratégies effectivement utilisées durant une tâche, et observer plusieurs fois un scripteur devant produire divers types de texte car probablement ses stratégies changent en fonction des écrits produits.

Dans le cas du second dispositif, l'absence de lien entre l'autorégulation et la performance peut renvoyer au fait que l'instrument utilisé n'intègre pas toutes les stratégies scripturales pouvant être employées de manière consciente par les étudiants interviewés. D'autres variables qui peuvent expliquer ces résultats sont le niveau des étudiants (leurs moyennes générales) et leur motivation. Cependant, la prise en compte de la première variable impliquerait, nous semble-t-il, des analyses poussées relativement au rapport entre la performance à l'écrit et la réussite aux études, ou entre cette dernière et l'autorégulation, ce qui n'était pas notre intérêt ici. Quant au rôle de la motivation, Vezin (1989) suggère que l'insuffisance d'investissement cognitif des sujets peut provoquer la non utilisation des connaissances métacognitives des sujets; pour cette raison, propose l'auteur, il résulte nécessaire de leur imposer des niveaux d'exigence élevés qui constituent des facteurs mobilisateurs des efforts métacognitifs. Cela signifie que, dans le cadre d'un dispositif de recherche, le chercheur puisse estimer préalablement le niveau et le type de motivation des sujets, et s'assurer des effets que son expérimentation peut avoir sur l'investissement de ces derniers. Bien que nous puissions considérer le facteur motivationnel comme une explication plausible, sa vérification mériterait des mesures dont il n'était pas question dans ce travail.

En outre, l'analyse des discours (ler dispositif) a permis de repérer quelques signes « d'expertise » en fonction de la classification de Bereiter $\&$ Scardamalia (1992). Par exemple, les étudiants reconnaissent les défis cognitifs de la production d'écrits. Ils identifient leurs faiblesses en tant que scripteurs, ainsi que les exigences textuelles d'une fiche de lecture et les attentes du lecteur. De cette manière, les étudiants interviewés tiennent compte non seulement des aspects formels de l'écrit mais aussi des exigences intellectuelles de l'écriture en ce qui concerne la transformation des connaissances et la production 
d'idées nouvelles. Ce résultat nous interpelle si nous pensons aux représentations de l'écriture que des étudiants ont révélées lors d'une étude menée par Delcambre \& Reuter (2000). Dans cette recherche, les étudiants n'évoquent que très peu la conception de l'écriture comme transformation de la pensée. Contrairement aux entretiens menés par ces auteurs, les étudiants participant à notre recherche ont déclaré des connaissances relatives à une situation d'écriture précise dans laquelle ils semblent avoir reçu des consignes précises de la part de l'enseignant. On peut penser que le fait de parler d'une tâche particulière, qui aurait permis une meilleure compréhension des contraintes de l'écriture, pourrait faciliter la reconnaissance de cette dimension de l'écriture nommée « épistémique » par Bereiter (1980).

Nonobstant, bien que les sujets reconnaissent ces défis de la production d'écrits, l'analyse de leurs stratégies d'autorégulation montre une quantité plus importante d'indices que nous pouvons appeler de non-expertise en nous appuyant sur les travaux de Zimmerman \& Kitsantas (1997). Ainsi, quant à la stratégie de fixation d'objectifs, les étudiants interrogés se sont plutôt focalisés sur la surveillance des caractéristiques des écrits (le contenu, la longueur, le choix de mots, la syntaxe), accordant ainsi moins d'efforts à l'observation des stratégies mises en œuvre au cours de leurs productions. Également, leurs critères d'évaluation et leurs verbalisations subvocales portent essentiellement sur les résultats à obtenir en termes de forme de l'écrit, et négligent la gestion des processus en cours. Un autre élément qui n'apparaît pas dans la description de leurs stratégies est la manière dont les étudiants abordent la dimension heuristique de l'écriture. Leurs connaissances révèlent qu'ils reconnaissent l'exigence de produire des nouvelles connaissances, de transformer des informations à partir des lectures d'ouvrages; mais leurs déclarations ne montrent pas la manière dont ils ont essayé de répondre à cette attente.

Comme nous l'avons évoqué, d'après Zimmerman (1998), Zimmerman \& Kitsantas (1999) Zimmerman $\&$ Risemberg (1997), le fait de contrôler en priorité les aspects de contenu et de forme ne contribue pas à une démarche efficace chez les non experts. En se fixant en priorité des buts relatifs aux stratégies à utiliser et à la manière de produire les résultats visés, ces rédacteurs réussiraient à mieux contrôler leurs démarches. C'est seulement après avoir atteint ce premier niveau de maîtrise qu'ils pourraient se centrer davantage sur l'accomplissement des critères de qualité exigés, aussi bien formels que textuels et heuristiques. Selon Piolat \& Roussey (1991b) le scripteur doit être capable de contrôler non seulement les aspects relatifs à la qualité du produit (éléments de surface et de fond) mais aussi les processus impliqués dans le processus d'écriture. Selon ces derniers auteurs, le sujet, en tant que scripteur proactif, peut prévoir le déroulement de sa production; puis, d'une manière réactive, il arrive à contrôler la qualité de son écrit vis-à-vis les normes et les buts fixés. Dans le cas des sujets de notre enquête, les buts et les critères explicités tiennent compte essentiellement des connaissances sur la tâche et ne mettent pas en lumière la relation entre ces informations et leurs connaissances stratégiques. Moins attentifs à la gestion des processus mis en place, ils ne semblent pas porter leur attention sur les meilleures manières de procéder ni de corriger leurs méthodes.

\section{Conclusion}

Nous venons de rendre compte des résultats d'une recherche qui avait un objectif double: décrire les stratégies d'autorégulation des étudiants et ce qu'ils connaissent d'eux-mêmes et de leurs manières d'écrire, d'une part; déterminer le rapport entre ces composants de la métacognition et la qualité des textes produits, d'autre part. Pour cela, nous avons mis en place une méthode qualitative, à l'aide d'entretiens d'explicitation, et une autre approche quantitative consistant en la passation d'un questionnaire. Dans le cadre de ces dispositifs, nous avons construit deux instruments de mesure. Une grille d'évaluation de textes nous a permis de déterminer la performance des étudiants à l'écrit telle qu'elle était considérée ici: la qualité des écrits. Puis, nous avons administré un questionnaire pour évaluer la métacognition à travers d'items qui explorent à la fois des connaissances métacognitives (sur la tâche, les stratégies et ses capacités de rédacteur) et certaines stratégies d'autorégulation (fixation de buts, autoévaluation et autoinstruction, entre autres).

Notre hypothèse principale qui supposait une dépendance forte et positive entre la métacognition et la performance à l'écrit est relativisée car il faut tenir 
compte du dispositif mis en place et de la dimension métacognitive dont il s'agit. Les liens entre ces variables sont plus forts lorsque le sujet s'exprime d'une manière decontextualisée que lorsqu'il évoque une situation précise. En se référant à une tâche particulière, le scripteur explicite une variété de stratégies dont le rapport avec la performance peut dépendre non seulement des caractéristiques de l'écrit mais aussi des compétences du scripteur luimême. Par ailleurs, il convient de souligner que des phénomènes de conscience ou d'oubli (ayant lieu au cours d'entretiens, par exemple) ont probablement empêché l'explicitation des méthodes employées dans la situation concrète et la description des stratégies les plus efficaces. La validité des données que nous avons présentées a pu être limitée également par les processus d'interprétation que le sujet fait de son expérience passée (entretiens) et/ou par la nonvéracité des réponses obtenue (entretiens et questionnaires). Nos conclusions ne se rapportent qu'aux déclarations des individus, raison pour laquelle nous insistons sur l'importance de mettre en place d'autres méthodologies telles que des observations directes qui seraient utiles pour repérer les stratégies que les sujets n'explicitent pas, et pour vérifier que les méthodes évoquées ont véritablement été mises en œuvre. Une reproduction de l'étude au moyen de questionnaires auprès d'une population plus large peut également être envisagée dans le but d'étendre la portée des résultats de ce premier travail.

En réponse à la question: quels sont les aspects métacognitifs les plus associés à la performance? cette recherche soulève la place centrale des connaissances métacognitives des étudiants relatives à leurs facilités/faiblesses pour écrire, à la tâche et à leurs stratégies. En fonction des déclarations des étudiants, l'obtention des meilleurs résultats à l'écrit est également liée à l'usage des connaissances métacognitives en vue de planifier l'écrit et de guider la production écrite. À partir des dispositifs déployés, nous constatons que les étudiants scripteurs qui ont montré une performance élevée ont été plus souvent ceux qui ont exprimé davantage de connaissances relatives à leurs méthodes, aux demandes et aux caractéristiques de la tâche, et ceux qui ont reconnu plus aisément leurs points faibles et forts en écriture.

Notre travail soulève l'idée que, dans les dispositifs d'aide aux étudiants, il serait souhaitable de rendre prioritaire la réflexion sur eux-mêmes et leurs méthodes afin de les amener, avant tout, vers une écriture réflexive qui s'appuie sur la prise de conscience de leurs processus et vers une connaissance approfondie des liens entre les caractéristiques de l'écrit à produire et leurs stratégies personnelles efficaces. Du point de vue pédagogique, plutôt que l'adoption d'un éventail de méthodes qui, à priori, seraient synonymes de la production de textes de qualité, notre travail confirme l'importance d'actions centrées sur le sujet plutôt que sur le produit à effectuer. Amener les étudiants à expliciter les exigences de la tâche, ainsi que leurs façons de les résoudre, peut les aider à porter une réflexion sur la pertinence de leurs connaissances, à mesurer leur efficacité par rapport aux objectifs donnés et à construire des nouvelles connaissances métacognitives. Ce serait à notre avis une des conditions de la didactique d'écriture centrée sur l'élève/étudiant, approche qui permettrait ainsi connaître au plus prés les processus et les connaissances du sujet pour informer ce dernier et, parallèlement, pour renseigner l'enseignant et le chercheur du fonctionnement des scripteurs, qu'ils soient élèves, étudiants ou adultes en formation. Ces objectifs nous renvoient vers des perspectives qui sont celles de plusieurs travaux (Boch, 1998; Pollet, 2001; Plane 2003; FiliapBaratte, 2004; Thyrion, 2004; Donahue, 2008; Delcambre \& Lahanier-Reuter, 2010) intéressés par l'analyse des représentations et des moyens dont disposent les élèves et les étudiants pour faire face aux contraintes de la production d'écrite en divers contextes scolaires et universitaires. 


\section{NOTES}

1. Pauron, A. (2001). La réussite en DEUG par université, session 1999. (Online), juillet 2006. Note d'information 01.47. Ministère de l'Éducation nationale, de l'Enseignement supérieur et de la Recherche, ftp://trf. education.gouv.fr/pub/edutel/dpd/ni0147.pdf

2. Girardot, P. et al. (2005). La réussite en licence en un an, deux et trois ans. Rapport de la DEP. Ministère de l'Éducation nationale, de l'Enseignement supérieur et de la Recherche, Paris, p. 48-61.

3. Girardot, P. et al. (2005), op. cit.

4. Rappelons que ces étudiants avaient produit une fiche de lecture dans le cadre de leurs études.

5. Pour obtenir une note finale sur 20 , à chaque item correspondaient des points. Ensuite, les valeurs de 0 à 3 renvoyaient chacun au $25 \%$ de ces points. Par exemple, si pour l'item 6 , qui valait 6,2 points, un étudiant cochait l'option 2, il obtenait 4.6 points pour sa réponse. En fonction des caractéristiques des écrits produits, nous avons attribué aux items 6, 5 et 4 respectivement les valeurs les plus élevées et, dans l'ordre, aux questions 3, 2, 7 et 1 les plus faibles.

6. Cf. procédure de notation du premier dispositif, annexe 1. Les items 5,6 et 8 avaient les valeurs les plus hautes. Puis les questions 7, 9 et 3 avaient des valeurs moyennes; enfin les items 1,2 et 4 portaient moins de points.

\section{RÉFÉRENCES}

Barré-De Miniac, C. (2000). Le rapport à l'écriture: aspects théoriques et didactiques. Paris: Presses universitaires du Septentrion.

Bereiter, C. (1980). Development in writing. In L. Gregg \& E. Steinberg (Eds.), Cognitive process in writing (p. 73-93). New Yersey: L. Elbaum associates.

Bereiter, C., Scardamalia, M. (1992). Dos modelos explicativos de los procesos de composición escrita. Infancia y aprendizaje, 58, 43-64.

Boch, F. (1988). Les pratiques de réécriture dans l'enseignement supérieur: profils groupaux. Lidil, 17, 57-64.

Boekaerts, M. (1999). Metacognitive experiences and motivational state as aspect of self-awareness: review and discussion. European journal of psychology of education, 14, 4, 571-584.

Brassart, D.-G. (2005). Didactique du français langue maternelle: approche(s) cognitive(s). In J.-L. Chiss, J. David, \& Y. Reuter (Eds.), Didactique du français, fondements d'une discipline (p. 95-118). Bruxelles: De Boeck.

Brossard, M. (1994). Quelques réflexions sur activités métalinguistiques et activités scolaires. Repères, 9, 29-36. 
Brown, A. (1987). Metacognition, executive control and other more misterious mechanismes. In F. Weinert $\&$ R. Kluwe (Eds.), Metacognition, motivation and understanding (p. 65-116). Hillsdale: Laurance Elbaum.

Coulon, A. (1997). Le métier d'étudiant: l'entrée dans la vie universitaire. Paris: PUF.

Coutelet, B., \& et Rouet, J.-F. (2004). Apprendre à chercher dans un texte: effets d'un entraînement à 8 et 10 ans, Enfance 4, 56, 357-386.

Donahue, C. (2008). Ecrire à l'université: analyse comparée en France et aux États-Unis. Villeneuve d'Ascq: Presses universitaires du Septentrion.

Delcambre, I., \& Lahanier-Reuter, D. (2009). Écrits et discipline dans l'université française: le cas des sciences humaines. In J.-M. Defays, A. Englebert, M.-C. Pollet, L. Rosier, \& F. Thyrion (Eds.), Acteurs et contextes des discours universitaires. Paris: L'Harmattan.

Escorcia, D. (2007). Composantes métacognitives et performance à l'écrit: une approche sociocognitive du travail étudiant. Thèse, Université Paris Ouest-Nanterre La Défense, Nanterre.

Fave-Bonnet, M.F., \& Clerc, N. (2001). Des héritiers aux nouveaux étudiants: 35 ans de recherche. Revue française de pédagogie, 136, 9-19.

Ferrari, M., Bouffard, T., \& Rainville, L. (1998). What makes a good writer? Differences in good and poor writers'self-regulation of writing. Instructional science, 26, 473-488.

Filiap-Baratte, M. (2004). Les étudiants, la lecture et l'écriture au long de la scolarité. Souvenirs d'étudiants en sciences de l'éducation. Communication présentée au 9e Colloque de l'AIRDF. Québec, Canada, Aout.

Fijalkow, J. (1995). Savoir lire: didactique déclarative, procédurale, contextuelle. Spirale, 15, 121-146.

Flavell, J. (1987). Speculations about the nature and development of metacognition. In F. Weinert \& R. Kluwe (Eds.), Metacognition, motivation and understanding (21-29). Hilsdale: LEA.

Flavell, J. (1992). Metacognition and Cognitive Monitoring: A new area of cognitive-developmental inquiry. In T. Nelson (Ed.), Metacognition: Core readings (p. 3-9). Boston: Allin and Bacon.

Flavell, J., Millet, P., \& Miller, S. (1993). Cognitive development. London: Prentice-Hall International.

Hadji, Ch. (1997). Lévaluation démystifiée. Paris: ESF editeur.

Galand, B., Neuville, S., \& Frenay, M. (2005). Léchec à l'université en communauté française belge: comprendre pour mieux prévenir. (Online), Septembre 2009. Les cahiers de la recherche en Éducation et Formation, 39, 5-17. http://www.girsef.ucl.ac.be/Cahiers_CFR/039.pdf.

Graham, S., Harris, K., \& Mason, L. (2005). Improving the writing performance knowledge, and self-efficacy of struggling young writers: The effects of self-regulated strategy development. Contemporary Educational Psychology, 30, 207-241.

Graham, S., Harris, K., \& Troia, G. (1998). Writing and self-regulation: cases from the self-regulated strategy development model. In B. Zimmerman \& D. Schunck (Eds.), Self-regulated learning: from teaching to selfreflective practice (p. 20-41). New York: The Guilford press. 
Gruel, L. (2003). Les étudiants jugent la vie étudiante. (Online), Mai 2008. OVE Infos. http://www.ove-national. education.fr/index.php?lang=fr $\&$ page $=$ oveinfos. php $\&$ id $=6$.

Hayes, J., \& Flower, L. (1980). Identifying the organization of writing processes. In L. Gregg \& E. Steinberg (Eds.), Cognitive processes in writing (p. 3-30). New Yersey: L. Elbaum associates.

Jarousse, J.-P., \& Michaut, C. (2001). Variété des modes d'organisation des premiers cycles et réussite universitaire. Revue française de pédagogie, 36, 41-51.

Mariné, C., \& Huet, N. (1998). Techniques d'autoévaluation de la métacognition: les mesures indépendantes de l'exécution de tâches. L'année psychologique, 98, 4, 711-726.

Maxwell, J. (1999). La modélisation de la recherche qualitative. Une approche interactive. Saint Paul Fribourg: Éditions Universitaires Fribourg Suisse.

Melot, A.M., \& Corroyer, D. (1992). Organisation of metacognitive knowledge: a condition for strategy use in memorisation. European journal of psychology of education, 1, 7, 23-38.

Mongeau, P., \& Hill, J. (1998). Relations entre l'explicitation, l'anticipation et la performance. Revue des sciences de l'éducation, 24, 2, 323-334.

Mucchieli, L. (1998). La pédagogie universitaire en question: le point de vue d'étudiants en premier cycle de psychologie. Recherche et formations, 29, 161-176.

Noël, B. (1991). La métacognition. Bruxelles: De Boeck Université.

Peronard, M. (2001). Comprensión de textos escritos y metacomprensión. (Online), Février 2008. http://educ. ar/educar/comprension-de-textos-escritos-y-metacomprension.html.

Pinard, A. (1992). Métaconscience et métacognition. Canadian Psyshology/Psychologie canadienne, 33, 1, 27-39.

Piolat, A., \& Roussey, J.-Y. (1991a). Narrative and descriptive text: revising strategies and procedures. European journal of psychology of education, 6, 2, 155-163.

Piolat, A., \& Roussey, J.-Y. (1991b). Stratégies experts de contrôle rédactionnel et définition de but. Repères, 4, 79-92.

Piolat, A., \& Roussey, J.-Y. (1996). Student's drafting strategies and text quality. Learning and instruction, 2, $111-129$

Plane, S. (2003). Stratégies de réécriture et gestion des contraintes d'écriture par des élèves de l'école élémentaire: ce que nous apprennent des écrits d'enfants sur l'écriture. Rivista Italiana de Psicolinguistica Applicata, $3,1,57-77$

Pollet, M.C. (2001). Pour une didactique des discours universitaires. Belgique: De Boeck Université.

Pommier, J.-L. (2005). La métacognition muette chez les bons élèves, un paradoxe? In M. Derycke \& E. Bautier (Eds.), Culture(s) et réflexivité (p. 73-84). Saint-Étienne: Presses universitaires de Saint-Étienne. 
Raphael, T., Englert, C., \& Kirschner, B. (1989). Students'metacognitive knowledge about writing. Research in the teachning of english, 23, 4, 343-378.

Romainville, M. (2002). L'évaluation des acquis des étudiants dans l'enseignement universitaire. (Online), Mai 2009. Rapport établi à la demande du Haut Conseil de l'évaluation de l'école, Paris. http://cisad.adc. education.fr/hcee/documents/rapport_Romainville. pdf.

Schraw, G. (2001). Promoting General Metacognitive Awareness. In H. Hartman (Ed.), Metacognition in learning and instruction (p. 3-16). Netherland: Kluwer Academic Publisher.

Schraw, G., \& Sperling-Dennison, R. (1994). Assessing metacognitive awareness. Contemporary educational psychology, 19, 460-475.

Thyrion, F. (2004). Les compétences d'écriture des étudiants en Lettres: rôle de l'enseignement reçu et indicateurs de professionnalisation. Communication présentée au $9^{e}$ Colloque de l'AIRDF, Québec, Canada, Aout.

Vermersch, P. (1994). L'entretien d'explicitation en formation initiale et continue. Paris: USF Editeur.

Vezin, J.-F. (1989). Autoévaluation de la compréhension de textes. European journal of psychology of education, $4,4,505-511$.

Victori, M. (1999). An analyses of writing knowledge in EFL composing: a case study of two effective and two less effective writers. System, 27, 537-555.

Zimmerman, B. (1998), Developing self-fulfilling cycles of academic regulation: an analysis of exemplary instructional models. In B. Zimmerman \& D. Schunk (Eds.), Self-regulated learning: from teaching to selfreflective practice (p. 1-19). New York: The Guilford press.

Zimmerman, B. (2002). Efficacité perçue et autorégulation des apprentissages durant les études: une vision cyclique. In P. Carré \& A. Moisan (Eds.), La formation autodirigée: aspects psychologiques et pédagogiques (p. 70-99). Paris: L'Harmattan.

Zimmerman, B., \& Kitsantas, A. (1997). Developmental phases in self-regulation: shifting from process goals to outcome goals. Journal of educational psychology, 89, 1, 29-36.

Zimmerman, B., \& Kitsantas, A. (1999). Acquiring writing revision skills: shifting process to outcome selfregulatory goals. Journal of educational psychology, 1, 29-36.

Zimmerman, B., \& Martinez-Pons M. (1986). Development of structured interview for assessing student use of self-regulated learning strategies. American educational research journal, 23, 4, 614-628.

Zimmerman, B., \& Martinez-Pons, M. (2004). Pursuing academic self-regulation: a 20 years methodological quest. In J. EE, A. Chang, \& O. Tan (Eds.), Thinking about the thinking: what educators need to know (p. 220-232). Singapore: Mc Graw hill. 


\section{ANNEXE 1. GRILLES D'ÉVALUATION D'ÉCRITS}

\section{Premier dispositif 6}

La présente grille a pour but de connaître le jugement que vous portez sur la qualité de cet écrit. Veuillez noter, dans chaque colonne, un chiffre de 0 à 3 selon votre niveau d'accord avec chaque affirmation.

\section{Utilisez l'échelle suivante:}

\begin{tabular}{|cccc|}
\hline 0 & 1 & 2 & 3 \\
\hline $\begin{array}{c}\text { Pas du tout } \\
\text { d'accord }\end{array}$ & $\begin{array}{c}\text { Un peu } \\
\text { d'accord }\end{array}$ & Plutôt d'accord & $\begin{array}{c}\text { Tout à fait } \\
\text { d'accord }\end{array}$ \\
\hline
\end{tabular}

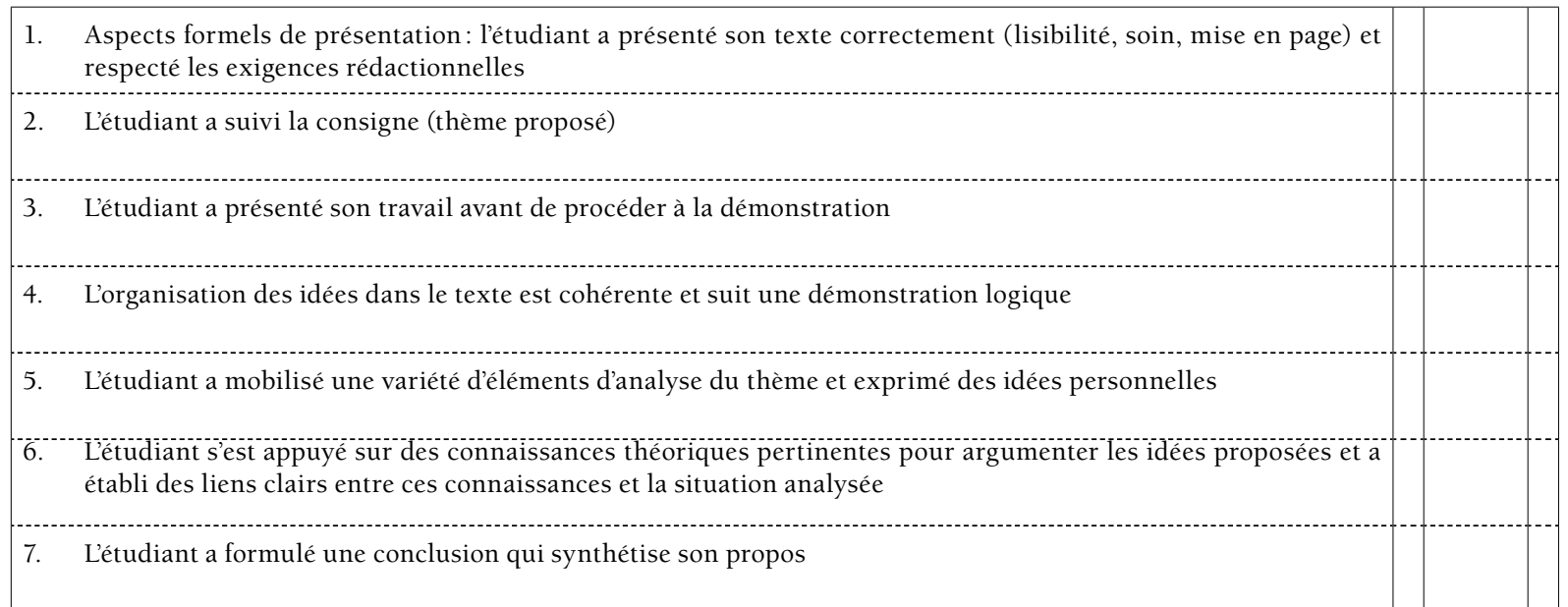




\section{Second dispositif ${ }^{7}$}

La présente grille a pour but de connaître le jugement que vous portez sur la qualité de cet écrit. Veuillez noter, dans chaque colonne, un chiffre de 0 à 3 selon votre niveau d'accord avec chaque affirmation.

\section{Utilisez l'échelle suivante:}

\begin{tabular}{|cccc|}
\hline 0 & 1 & 2 & 3 \\
\hline $\begin{array}{c}\text { Pas du tout } \\
\text { d'accord }\end{array}$ & $\begin{array}{c}\text { Un peu } \\
\text { d'accord }\end{array}$ & Plutôt d'accord & $\begin{array}{c}\text { Tout à fait } \\
\text { d'accord }\end{array}$ \\
\hline
\end{tabular}

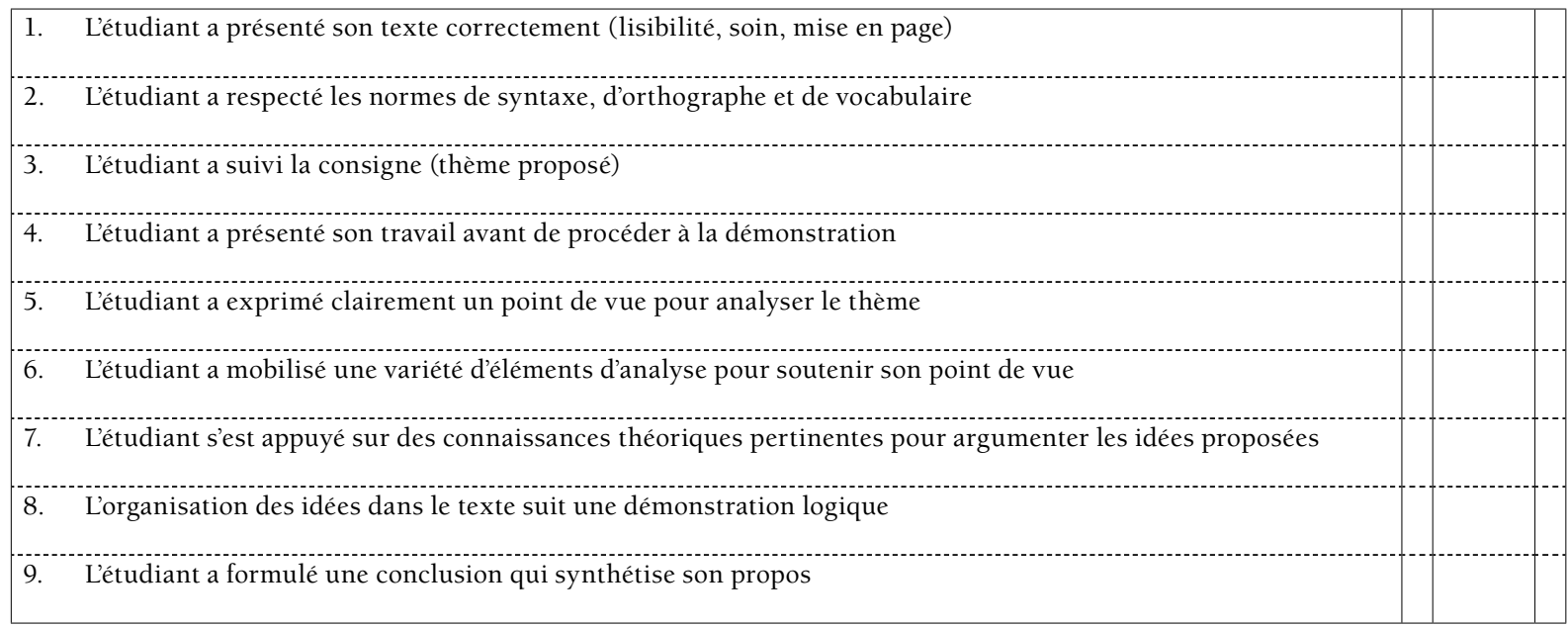




\section{Dyanne Escorcia}

\section{ANNEXE 2. QUESTIONNAIRE}

Age: Sexe: M F

Filière d'étude: Niveau d'étude

Nous souhaitons connaître la façon dont vous vous y prenez pour rédiger des écrits à domicile dans le cadre de vos études. Lisez attentivement les propositions ci-dessous et cochez un chiffre de 1 à 4 selon la réponse qui correspond le mieux à vos habitudes quand vous entreprenez un travail écrit. Suivez l'échelle suivante:

1 : jamais 2 : rarement 3 : fréquemment 4 : tout le temps

Prenez le temps pour répondre soigneusement à chaque question, vous disposez de 20 minutes. Ce questionnaire est confidentiel. Nous garantissons l'anonymat des données.

\section{Diriez-vous: "Quand je dois faire un travail écrit... "}

1. je prends en compte les objectifs de la tâche avant d'écrire

2. je sais quelles sont les caractéristiques du type d'écrit que je dois rédiger

3. je réfléchis à ce qui sera nécessaire d'écrire avant de commencer

4. je fais d'abord une liste de mots ou phrases clefs à utiliser pour aborder le thème

5. je sais organiser les informations dont je dispose avant de rédiger

6. j'ai du mal à reconnaître mes forces et faiblesses dans le domaine de l'écrit

7. je sais me motiver pour écrire

8. je suis un bon juge de la qualité de mon écrit

9. je commence à rédiger dès que j’ai lu la consigne

10. avant de commencer, je connais la structure du type de texte à rédiger

11. au début, je fais un plan en organisant les idées à traiter

12. j'utilise un guide méthodologique pour identifier les points à développer

13. lorsque j'utilise une méthode durant la rédaction, j'ai un objectif précis à l'esprit

14. quand j'écris, le lieu où je suis est secondaire

15. j’analyse l'efficacité de mes façons de faire pendant que j'écris

16. quel que soit l'objectif de mon travail, j'utilise toujours les mêmes méthodes

17. je me base exclusivement sur mon point de vue quant à la qualité de mon texte

18. je prends conscience des difficultés du type d'écrit seulement quand je suis en train de rédiger

19. pendant la rédaction, je me dis à haute voix ou mentalement ce que je vais faire

20. j'estime la longueur de mon écrit en cours de rédaction

21. j'évalue la qualité de mon écrit uniquement après avoir fini d'écrire

\begin{tabular}{|c|c|c|c|}
\hline 1 & 2 & 3 & 4 \\
\hline 1 & 2 & 3 & 4 \\
\hline 1 & 2 & 3 & 4 \\
\hline 1 & 2 & 3 & 4 \\
\hline 1 & 2 & 3 & 4 \\
\hline 1 & 2 & 3 & 4 \\
\hline 1 & 2 & 3 & 4 \\
\hline 1 & 2 & 3 & 4 \\
\hline 1 & 2 & 3 & 4 \\
\hline 1 & 2 & 3 & 4 \\
\hline 1 & 2 & 3 & 4 \\
\hline 1 & 2 & 3 & 4 \\
\hline 1 & 2 & 3 & 4 \\
\hline 1 & 2 & 3 & 4 \\
\hline 1 & 2 & 3 & 4 \\
\hline 1 & 2 & 3 & 4 \\
\hline 1 & 2 & 3 & 4 \\
\hline 1 & 2 & 3 & 4 \\
\hline 1 & 2 & 3 & 4 \\
\hline 1 & 2 & 3 & 4 \\
\hline 1 & 2 & 3 & 4 \\
\hline
\end{tabular}

